\title{
Selenoprotein L-inspired nano-vesicular peroxidase mimics based on amphiphilic diselenides
}

Wataru Uehara ${ }^{\text {a }}$, Sakura Yoshida ${ }^{\text {a }}$, Yui Emaya ${ }^{a}$, Takeshi Fuchigami ${ }^{a}$,

Mamoru Haratake ${ }^{b, *}$, Morio Nakayama ${ }^{a,{ }^{* *}}$

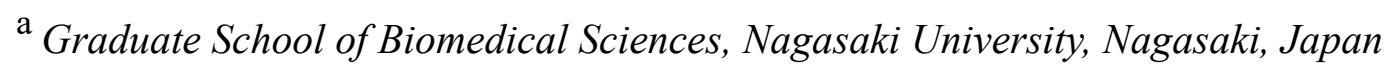

${ }^{\mathrm{b}}$ Faculty of Pharmaceutical Sciences, Sojo University, Kumamoto, Japan

* Corresponding author at: Faculty of Pharmaceutical Sciences, Sojo University, 4-22-1 Ikeda, Kumamoto 860-0082, Japan.

** Corresponding author at: Graduate School of Biomedical Sciences, Nagasaki University, 1-14 Bunkyo-machi, Nagasaki, 852-8521 Japan.

E-mail addresses: haratake@ph.sojo-u.ac.jp (M. Haratake),morio@nagasaki-u.ac.jp (M. Nakayama) 


\section{A B S T R A C T}

In this study, we developed selenoprotein L-inspired nano-vesicular peroxidase mimics based on amphiphilic diselenides. Selenocystine (SeCyst) was used as the starting material for the synthesis of four liposomal membrane-compatible diselenide derivatives ( $\mathrm{R}-\mathrm{Se}-\mathrm{Se}-\mathrm{R}$ ') with two hydrophobic tails and a polar part. The diselenide derivatives were successfully incorporated into the phosphatidylcholine (PC)-based nano-vesicular scaffold. The results of the particle diameter and zeta-potential measurements suggested that the functional diselenide moiety was placed around the outer surface, not in the hydrophobic interior, of the liposomal membrane structures. The GPx-like catalytic activity of the diselenide / PC liposomes was determined by the conventional NADPH method using glutathione as the reducing substrate. For three peroxide substrates, i.e., hydrogen peroxide, organic tert-butyl hydroperoxide and cummen hydroperoxide, the cationic property-possessing diselenide derivatives in the PC-based liposomes resulted in a higher catalytic activity in comparison to electrically neutral and anionic derivatives. Overall, the diselenide derivatives at the surface of a liposomal colloidal scaffold could exert a GPx-like catalytic activity in physiological aqueous media.

Keywords: diselenide; liposome; peroxidase; selenium; selenoprotein L.

Abbreviations: Cys, L-cysteine; DAN, 2,3-diaminonaphtalene; GPx, glutathione peroxidase; GSH, glutathione in the reduced form; MALDI TOF-MS, matrix-assisted laser desorption ionization time of flight-mass spectrometry; NADPH, nicotinamide adenine dinucleotide phosphate; PC, hydrogenated egg yolk phosphatidylcholine; SeCys, Sec and U, seleno-L-cysteine; H-SeCyst-OH, seleno-L-cystine; SelL, selenoprotein L; TBTU, 2-(1H-benzotriazol-1-yl)-1,1,3,3-tetramethyluronium tetrafluoroborate; TEA, triethylamine; $p$-TSA, $p$-toluenesulfonic acid; XPS, X-ray photoelectron spectroscopy. 


\section{Introduction}

The element selenium belongs to chalcogens in the periodic table, together with oxygen, sulfur and tellurium. After its discovery in the early 19th century, selenium was long considered as a solely poison until it was recognized as a nutrient for bacteria, birds and mammals in the mid 20th century [1]. Nutritional selenium is known to be utilized with diverse chemical forms by biomolecules. The representative chemical form of selenium, called selenocysteine (SeCys), is the 21st amino acid in the natural proteinogenic repertoire. As SeCys is encoded by the UGA termination codon that normally serves as a stop signal [2], its insertion sequence elements (SECIS) are a fundamental aspect of the mRNA encoding selenoproteins in eukaryotes [3]. The SECIS mechanism is utilized for the biosynthesis of 25 selenoproteins in humans; glutathione peroxidases (GPxs), thioredoxin reductase, iodothyronine deiodinases, selenophosphate synthase, selenoprotein P, etc. [4]. In nature, this SeCys insertion process is highly regulated, however, it is quite difficult to prepare selenoproteins using the recombinant expression systems [5].

The human selenoproteins, except for selenoprotein P, have a single SeCys residue (Sec) in their polypeptide chains. Sec is a functional element of the active site of the antioxidative enzyme GPxs. GPxs utilize catalytically-active selenol $(-\mathrm{SeH})$ of Sec to convert hydrogen peroxide and lipid peroxides into water and the corresponding alcohols using glutathione $(\mathrm{GSH})$ as the reducing substrate $\left(\mathrm{R}-\mathrm{OOH}+2 \mathrm{GSH} \rightarrow \mathrm{R}-\mathrm{OH}+\mathrm{GSSG}+\mathrm{H}_{2} \mathrm{O}\right)$. Several other selenoproteins are also oxidoreductases containing the catalytic redox-active Sec [6]. Such selenoenzymes can be typically a thousand times more effective in catalysis than their cysteine residue (Cys) homologs in place of Sec [7]; for instance, the rate of thioredoxin reduction increases up to $\approx 10^{2}-10^{4}$-fold by the seleno-glutaredoxin 3 analogues [8]. The kinetic and thermodynamic properties of the selenol group are also distinctly different from those of the thiol group. Sec in many selenoenzymes forms transient selenenylsulfide bonds $(-\mathrm{Se}-\mathrm{S}-)$ with proximal $\mathrm{Cys}$ as part of their catalytic mechanisms. Oxidoreductases containing either selenenylsulfide or diselenide $\left(-\mathrm{Se}^{-} \mathrm{Se}-\right)$ bonds can have physiologically compatible redox potentials and enhanced reduction kinetics in comparison to their sulfide counterparts. 
Marine animals have more selenoproteins than terrestrial animals. The selenoproteomes of fish are greater in number than those of mammals; with 30-37 selenoproteins, the selenoproteomes of fish are among the highest known [9]. The same core selenoprotein families are found in mammals and fish. In addition, fish have several species-specific selenoproteins [fish $15 \mathrm{kDa}$ selenoprotein-like protein, selenoprotein $\mathrm{J}$ and selenoprotein L (SelL)] that are missing in mammals [9]. Among the fish-specific selenoproteins, SelL is found in diverse aquatic organisms, including fish, invertebrates, and marine bacteria [10]. This selenoprotein uniquely contains two Sec separated by two other residues to form a $-\mathrm{Sec}-\mathrm{Xaa}-\mathrm{Xaa}-\mathrm{Sec}-$ motif (two Sec residues separated by two Xaa ones, Fig. 1a) similar to the catalytic $-\mathrm{Cys}-\mathrm{Xaa}-\mathrm{Xaa}-\mathrm{Cys}-$ motif (two Cys residues separated by two Xaa ones) in the thiol/disulfide oxidoreductase of thioredoxin (Trx), which suggests the redox function of this selenoprotein.

Low-molecular-mass organoselenium compounds have been reported as GPx mimics. They mostly do not exert a catalytic activity in physiological aqueous media because of their poor water-compatibility. Therefore, it is very difficult to apply them to medicinal applications. Most selenium-containing small molecules exhibit a low GPx-like catalytic activity because the catalytically-active selenol is chemically unstable, and there is not good way of generating the selenol. We have reported a nano-structured GPx mimic using the diselenide-containing SeCyst derivatives conjugated with the polysaccharide pullulan, which improves the chemical stability of selenium atoms, and hence, increases the GPx-like catalytic activity in comparison to the free SeCyst [11]. We also reported that another GPx mimic using a membrane-compatible selenenylsulfide derivative, which shows the GPx-like catalytic activity comparable to the SeCyst-pullulan conjugate [12]. In this study, we developed a diselenide-containing peroxidase nano-mimic based on a molecular assembly of which the membrane-compatible amphiphilic diselenide derivatives were reconstituted into a liposomal membrane. 


\section{Materials and methods}

\subsection{Materials}

Seleno-L-cystine (H-SeCyst-OH) was obtained from Sigma Co., Ltd. (St. Louis, MO). $n$-Hexadecanoic acid and 2-(1H-benzotriazol-1-yl)-1,1,3,3-tetramethyluronium tetrafluoroborate (TBTU) were obtained from Nacalai Tesque, Inc. (Kyoto, Japan). 2,3-Diaminonaphthalene (DAN) and glutathione in the reduced form (GSH) were obtained from Tokyo Chemical Industry Co., Ltd. (Tokyo, Japan). Hydrogenated egg yolk phosphatidylcholine (PC) was obtained from the NOF Corporation (Tokyo, Japan). GSH reductase and nicotinamide adenine dinucleotide phosphate in the reduced form (NADPH) were obtained from Wako Pure Chemical Ind., Ltd. (Osaka, Japan). Hydrogen peroxide, cumene hydroperoxide and tert-butyl hydroperoxide used as the substrates for the GPx-like catalytic activity measurements were purchased from Nacalai Tesque, Inc. A Milli-Q Biocel system (Millipore Corp., Billerica, MA) was utilized to generate water (> $18 \mathrm{M} \Omega \square \mathrm{cm}$ ), which was used throughout this study. All other chemicals were of commercial reagent or special grades and used as received.

\subsection{Synthesis of diselenide derivatives}

H-SeCyst-OD: A mixture of H-SeCyst-OH (133.6 mg, $0.4 \mathrm{mmol})$, p-toluenesulfonic acid (p-TSA) (189 mg, $1 \mathrm{mmol})$ and $n$-dodecanol $(178 \mu \mathrm{L}, 0.8 \mathrm{mmol})$ was suspended in toluene $(30 \mathrm{~mL})$, then refluxed for $40 \mathrm{~h}$. After removal of the toluene using a rotary evaporator, the resulting precipitate was washed several times with saturated $\mathrm{NaHCO}_{3}$ and/or $\mathrm{NaCl}$, then extracted into $\mathrm{CHCl}_{3}$. The solvent was removed under reduced pressure (yield 66.7\%). ${ }^{1} \mathrm{H}$ NMR [a Varian Gemini 300 MHz spectrometer (Agilent Technologies Inc., Santa Clara, CA), $\left.\mathrm{CDCl}_{3}\right]: \delta 0.86-0.90(\mathrm{t}, 6 \mathrm{H}, J=6.6 \mathrm{~Hz}), 1.21-1.31(\mathrm{~m}, 32 \mathrm{H}), 1.52-1.68(\mathrm{~m}, 8 \mathrm{H}), 3.16-3.38$ (m, 4H), 3.76-3.78 (dd, 2H, $J=7.65,12.6 \mathrm{~Hz}), 4.11-4.16(\mathrm{t}, 4 \mathrm{H}, J=6.9 \mathrm{~Hz})$, MALDI TOF-MS [an Ultraflex III (Bruker Daltonics, Bremen, Germany), positive ion mode]: calcd for $\mathrm{C}_{30} \mathrm{H}_{60} \mathrm{~N}_{2} \mathrm{O}_{4}{ }^{80} \mathrm{Se}_{2} 672.82$, found $m / z 672.80$. 
Ac-SeCyst-OD: H-SeCyst-OH (120 mg, $0.18 \mathrm{mmol}$ ) was dissolved in $\mathrm{CH}_{2} \mathrm{Cl}_{2}$ containing $\mathrm{N}\left(\mathrm{CH}_{3}\right)_{3}$, then $\mathrm{CH}_{3} \mathrm{COCl}(28 \mu \mathrm{L}, 0.4 \mathrm{mmol})$ was added to the mixture in an ice-cold bath. The mixture was stirred overnight at room temperature. After removal of the solvent, the resulting precipitate was washed with saturated $\mathrm{NaHCO}_{3}$, then dissolved in an appropriate volume of $\mathrm{CHCl}_{3}$ containing anhydrous $\mathrm{Na}_{2} \mathrm{SO}_{4}$ (yield 66.7\%). ${ }^{1} \mathrm{H}$ NMR $(300 \mathrm{MHz}$, $\left.\mathrm{CDCl}_{3}\right): \delta 0.82-0.90(\mathrm{t}, 6 \mathrm{H}, J=15.2 \mathrm{~Hz}), 1.41-1.18(\mathrm{~m}, 32 \mathrm{H}), 2.26(\mathrm{~s}, 6 \mathrm{H}), 4.07-4.03(\mathrm{t}, 4 \mathrm{H}$, $J=6.8 \mathrm{~Hz}), 4.15-4.10(\mathrm{~m}, 2 \mathrm{H})$, ESI TOF-MS [a JMS-T100LC (JEOL, Tokyo, Japan), positive ion mode]: calcd for $\mathrm{C}_{34} \mathrm{H}_{64} \mathrm{~N}_{2} \mathrm{O}_{4}{ }^{80} \mathrm{Se}_{2} 756.89$, found $m / z 779.15$.

D-SeCyst-OMe: A mixture of H-SeCyst-OH (334 mg, $1 \mathrm{mmol})$ and $p$-TSA (3 g, 15.7 mmol) dissolved in $10 \mathrm{~mL}$ of absolute $\mathrm{CH}_{3} \mathrm{OH}$ was refluxed for $40 \mathrm{~h}$. After removal of the alcohol, the resulting precipitate was left for $24 \mathrm{~h}$ in $\mathrm{C}_{2} \mathrm{H}_{5} \mathrm{OC}_{2} \mathrm{H}_{5}$. The obtained yellow solid material was washed several times with $\mathrm{C}_{2} \mathrm{H}_{5} \mathrm{OC}_{2} \mathrm{H}_{5}$, then dried under reduced pressure (yield 90.5\%). The obtained H-SeCyst-OMe (36.2 mg, $0.1 \mathrm{mmol})$ and $n$-dodecanoic acid (44 mg, $0.22 \mathrm{mmol})$ were dissolved in $\mathrm{CH}_{3} \mathrm{CN}(10 \mathrm{~mL})$, then TBTU $(64.2 \mathrm{mg}, 0.2 \mathrm{mmol})$ and $\mathrm{N}\left(\mathrm{C}_{2} \mathrm{H}_{5}\right)_{3}(60 \mu \mathrm{L}, 0.43 \mathrm{mmol})$ were added in this solution. The mixture was stirred overnight at room temperature. After removal of the solvent, the resulting precipitate was washed with saturated $\mathrm{NaHCO}_{3}$, then dissolved in an appropriate volume of $\mathrm{CHCl}_{3}$ containing anhydrous $\mathrm{Na}_{2} \mathrm{SO}_{4}$. The obtained yellow oil material was washed several times with $\mathrm{CHCl}_{3}$, then dried under reduced pressure (yield 74.3\%). ${ }^{1} \mathrm{H}$ NMR $\left(300 \mathrm{MHz}, \mathrm{CDCl}_{3}\right): \delta 0.86(\mathrm{t}, 6 \mathrm{H}$, $J=6.6 \mathrm{~Hz}), 1.26-1.30(\mathrm{~m}, 32 \mathrm{H}), 1.53(\mathrm{~m}, 4 \mathrm{H}), 1.80-2.10(\mathrm{~m}, 4 \mathrm{H}), 3.66(\mathrm{~s}, 6 \mathrm{H}), 4.40(\mathrm{~m}, 2 \mathrm{H})$, MALDI TOF-MS (an Ultraflex III, positive ion mode): calcd for $\mathrm{C}_{32} \mathrm{H}_{64} \mathrm{~N}_{2} \mathrm{O}_{6}{ }^{80} \mathrm{Se}_{2} 728.84$, found $m / z 728.89$.

D-SeCyst-OH: H-SeCyst-OH (334 mg, $1 \mathrm{mmol}$ ) was suspended in tert-butyl acetate (50 $\mathrm{mL})$ and perchloric acid $(0.8 \mathrm{~mL})$. After stirring overnight at room temperature, the mixture was subsequently added to saturated $\mathrm{NaHCO}_{3}$ and extracted into $\mathrm{CH}_{3} \mathrm{COOC}_{2} \mathrm{H}_{5}$ containing anhydrous $\mathrm{Na}_{2} \mathrm{SO}_{4}$. The resulting $\mathrm{H}-\mathrm{SeCyst}-\mathrm{O} t \mathrm{Bu}(56 \mathrm{mg}, 0.125 \mathrm{mmol})$ and $n$-dodecanoic acid $(55 \mathrm{mg}, 0.275 \mathrm{mmol})$ were dissolved in $\left(\mathrm{CH}_{3}\right)_{2} \mathrm{NCHO}$, then stirred overnight at room temperature. After removal of the $\left(\mathrm{CH}_{3}\right)_{2} \mathrm{NCHO}$ under reduced pressure, the crude material was sequentially washed several times with $10 \%$ citric acid and saturated $\mathrm{NaCl}$, and dried with $\mathrm{Na}_{2} \mathrm{SO}_{4}$. The resulting material was dissolved in $\mathrm{CH}_{2} \mathrm{Cl}_{2}$ with $\mathrm{CF}_{3} \mathrm{COOH}$ and stirred 
overnight at room temperature, then dried under reduced pressure (yield 70.2\%). ${ }^{1} \mathrm{H}$ NMR $\left(300 \mathrm{MHz}, \mathrm{CDCl}_{3}\right): \delta: 0.86-0.92(\mathrm{t}, 6 \mathrm{H}, J=12 \mathrm{~Hz}), 1.27-1.31(\mathrm{~m}, 32 \mathrm{H}), 1.51-1.58(\mathrm{~m}, 4 \mathrm{H})$, 1.80-2.09 (m, 4H), 4.52 (m, 2H), ESI TOF-MS (a JMS-T100LC, positive ion mode): calcd for $\mathrm{C}_{30} \mathrm{H}_{60} \mathrm{~N}_{2} \mathrm{O}_{6}{ }^{80} \mathrm{Se}_{2} 700.79$, found $m / z$ 701.16.

\subsection{Preparation of diselenide/PC liposomes}

$\mathrm{PC}$ and diselenide at the indicated molar ratio were mixed in a $100-\mathrm{mL}$ round-bottom flask and dissolved by adding $5 \mathrm{~mL}$ of $\mathrm{CHCl}_{3}$. The $\mathrm{CHCl}_{3}$ was then gently removed. After the addition of $50 \mathrm{~mL}$ of $10 \mathrm{mM}$ Tris/ $\mathrm{HCl}$ buffer $(\mathrm{pH} \mathrm{7.4)}$ to the flask, the mixture was vortexed for $1 \mathrm{~h}$. The resulting suspension was subsequently sonicated using a Sonifier 250D probe-type sonicator (Branson, Danbury, CT) at $80 \mathrm{~W}$ for $15 \mathrm{~min}$ in an ice-water bath. The opaque liposomal suspensions were then centrifuged at $40000 \mathrm{rpm}$ for $1 \mathrm{~h}$. The supernatant was used in subsequent experiments as the liposomal diselenide derivative/PC solution.

\subsection{Determination of particle diameter and zeta-potential of the liposomes}

The particle diameter and distribution of the liposomes were measured by a dynamic light scattering (DLS) technique using a Zetasizer Nano ZS (Malvern Instruments, Ltd., Worcestershire, UK) at $90^{\circ}$ to the incident beam (a $633 \mathrm{~nm}$ beam from a $4 \mathrm{~mW}$ He-Ne laser tube) in a 1-cm pass-length quartz cell at $25{ }^{\circ} \mathrm{C}$. Data fitting was carried out using a multimodal algorithm supplied by Malvern Instruments. The obtained correlograms were fitted to diffusion coefficients and converted to a hydrodynamic diameter using the Einstein-Stokes equation. The zeta-potential of the liposomes was determined by a laser Doppler method using an ELS-7500 (Otsuka Electronics Co., Ltd., Osaka, Japan) in a DTS1070 capillary cell.

\subsection{Determination of phosphorus concentration}


After acid digestion of the lipid vesicle suspensions using a 4:1 mixture by volume of $\mathrm{HNO}_{3}$ and $\mathrm{HClO}_{4}$, the phosphorus content was determined based on vanadate, which can generate a yellow complex with the phosphate ion [13]. The color produced by the complexation was monitored at $440 \mathrm{~nm}$. The amount of phosphorus in the liposomes was calculated by the difference in the phosphorus concentration of the liposomal solution after centrifugation.

\subsection{Determination of selenium concentration}

The selenium concentrations in the specimens were determined by fluorometry using DAN after acid digestion using a 4:1 mixture by volume of $\mathrm{HNO}_{3}$ and $\mathrm{HClO}_{4}$ [14]. The selenium standard solution [1000 ppm as selenium (IV) dioxide in $0.1 \mathrm{M} \mathrm{HNO}_{3}$ ] for the fluorometry was obtained from Kanto Chemical Co., Inc.

\subsection{X-ray photoelectron spectroscopy}

According to previously reported procedures [14,15], X-ray photoelectron spectroscopy (XPS) was performed using an AXIS-ULTRA instrument fitted with a delay-line detector and a monochromated Al $K \alpha$ line $(0.8339 \mathrm{~nm}, 1.486 \mathrm{keV})$ operating at $10 \mathrm{kV}$ and $13 \mathrm{~mA}$ (Shimadzu Corp., Kyoto, Japan).

\subsection{Determination of GPx-like catalytic activity}

The GPx-like catalytic activity was determined according to previously reported procedures $[11,12,16]$. The liposome solution (final selenium concentration: $5 \mu \mathrm{M}$ ) was sequentially combined with ethylenediaminetetraacetic acid (1 mM), $\mathrm{NaN}_{3}(1 \mathrm{mM}), \mathrm{GSH}$ reductase (1 unit), GSH (1 mM), and NADPH $(0.25 \mathrm{mM})$ in $10 \mathrm{mM}$ Tris/HCl buffer $(\mathrm{pH} 7.4)$. The reaction was initiated by the addition of a peroxide substrate $\left(\mathrm{H}_{2} \mathrm{O}_{2}\right.$, cumene hydroperoxide, tert-butyl hydroperoxide) solution $(0.5 \mathrm{mM})$. The optical density (OD) at $340 \mathrm{~nm}$ due to the NADPH was recorded every $10 \mathrm{~s}$ just after mixing by inversion. The 
GPx-like catalytic activity was calculated using eq. 1 as micromoles of NADPH oxidized per minute.

$$
\text { GPx-like catalytic activity }=\left(\Delta \mathrm{OD}_{\mathrm{smp}}-\Delta \mathrm{OD}_{\mathrm{blk}}\right) \times 1000 / \varepsilon_{\mathrm{mM}} / \mathrm{c} \cdots \cdots(1)
$$

where $\Delta \mathrm{OD}_{\mathrm{smp}}$ is the decrease in $\mathrm{OD}$ at $340 \mathrm{~nm}$ of the sample solutions between 10 and $190 \mathrm{~s}$ after the addition of the peroxide substrate, $\triangle \mathrm{OD}$ blk is the decrease in OD at $340 \mathrm{~nm}$ per minute of the solutions without the liposome, $\varepsilon \mathrm{mM}$ is the extinction coefficient for the $1 \mathrm{mM}$ NADPH solution $[6.22 /(\mathrm{mM} \cdot \mathrm{cm})]$, and $\mathrm{c}$ is the final selenium concentration in $\mu \mathrm{M}[17,18]$. The primary data of the absorbance changes in the experiments of the activity measurement were listed in Table 1S in Supplementary materials. 


\section{Results and discussion}

Selenocystine (SeCyst) was used as the starting material for the synthesis of four membrane-compatible diselenide derivatives ( $\mathrm{R}-\mathrm{Se}-\mathrm{Se}-\mathrm{R}$ ') with two hydrophobic tails and a polar part (Scheme 1). SeCyst was coupled with $n$-dodecanoic acid or $n$-dodecanol through either its $\alpha$-amino or $\alpha$-carboxyl groups. In order to determine the effect of the ionic property in the vicinity of the diselenide moiety on the GPx-like catalytic activity, a free $\alpha$-amino group-possessing $\mathrm{H}$-SeCyst-OD and a free $\alpha$-carboxyl group-possessing D-SeCyst-OH were synthesized. Ac-SeCyst-OD and D-SeCyst-OMe were also derivatized by $\mathrm{N}$-acetylation and methyl esterification, respectively. Their amphiphilic structures are thought to be compatible to the phospholipid-based liposomal membrane in a proper molecular orientation. Selenium contains six stable isotopes $\left({ }^{74} \mathrm{Se},{ }^{76} \mathrm{Se},{ }^{77} \mathrm{Se},{ }^{78} \mathrm{Se},{ }^{80} \mathrm{Se}\right.$ and ${ }^{82} \mathrm{Se}$ ) and their natural abundances are $0.89,9.37,7.63,23.77,49.61$ and 8.73 atom\%, respectively. Therefore, selenium atom-containing molecules are known to provide a characteristic isotope mass spectral pattern. The distinctive selenium isotope patterns of the diselenide derivatives containing two selenium atoms were clearly observed in their mass spectra (Fig. 2). The values of $\mathrm{m} / \mathrm{z}$ and isotope pattern for H-SeCyst-OD and D-SeCyst-OMe and D-SeCyst-OH were in good agreement with the calculated values. An almost identical isotope pattern for Ac-SeCyst-OD was detected as its sodium adduct.

To characterize the oxidation and chemical bond states of the selenium atoms, the diselenide derivatives were analyzed by X-ray photoelectron spectroscopy (XPS). XPS measures the elemental composition in the chemical state and electronic state of the sample surfaces by X-ray radiation. The XPS spectra are obtained by irradiating the materials with $\mathrm{X}$-rays while measuring the number of electrons and the binding energy. Both the elements and electronic orbitals have specific relationships to the binding energy from the diselenide bond. The obtained absorption spectra and estimated values for the binding energy of the diselenide bond are indicated in Fig. S1 and Table 1, respectively. The selenium $3 p$ and $3 d$ electrons from SeCyst used as the starting material gave three absorption peaks at 166.1 for selenium 3p $3 / 2$, 166.7 for selenium 3 $\mathrm{p}_{1 / 2}$ and $54.9 \mathrm{eV}$ for selenium $3 \mathrm{~d}$ (Fig. S1e). Meanwhile, the absorption peak shapes of the four-diselenide derivatives were almost identical to those of 
SeCyst. The selenium $3 p$ and $3 d$ electrons from the diselenide derivatives provided distinctive absorption peaks at around 55, 161 and $167 \mathrm{eV}$. When compared to elemental selenium [Se(0)], selenious acid [Se(IV)] and selenic acid [Se(VI)], no absorption peaks assigned to such oxidized selenium species were detected in the XPS survey spectra of the diselenide derivatives. Therefore, the values for the binding energy of the selenium $3 p$ and $3 \mathrm{~d}$ components of the four-diselenide derivatives were almost equal to those of SeCyst, and all the derivatives were thought to mostly exist in the oxidative state number of minus 1 .

Therefore, the sulfur and selenium atoms in the OHSeP molecule were thought to form the selenenylsulfide linkage and to mostly exist in the oxidation state (number) of -1 .

Due to their poor water-solubility, most of the low-molecular-mass GPx mimics previously reported are hardly applicable to medicinal use. We have already tried to develop water-compatible GPx mimics that can exert the GPx-like catalytic activity in physiological aqueous media. In our previous paper, the lipid/water interface of the liposomal membranes was reported to provide an effective colloidal scaffold for the development of water-soluble GPx nano-mimics [12]. The functional diselenide moiety could possibly be oriented toward the bulk water phase at the outer surface of the liposomal membrane, which allows reducing substrate thiol compounds to nucleophilically attack the selenium atoms and subsequent generation of the catalytically-active selenol groups that exert the key role in the GPx-like catalytic activity (Fig. 1b). The liposomes were prepared by the Banghan method from a mixture of the diselenide derivative and hydrogenated egg yolk phosphatidylcholine (PC) in a given molar ratio $(20: 80 \mathrm{~mol} \%)$.

The particle diameters of the diselenide/PC liposomes were determined by the dynamic light scattering technique (Table 2). The mean particle diameters of the diselenide-free PC liposome used in this study were $94.5 \pm 0.466 \mathrm{~nm}$. The H-SeCyst-OD/PC liposome gave rise to a higher value in comparison to the PC liposome of $136 \pm 17.1 \mathrm{~nm}$. The particle diameters of the Ac-SeCyst-OD/PC and D-SeCyst-OMe/PC liposomes were almost similar to each other $(76.0 \pm 21.4$ and $73.3 \pm 14.1 \mathrm{~nm})$, and slightly lower than that of the PC liposome. The D-SeCyst-OH/PC liposome was somewhat smaller than the other liposomes, probably due to the ionic character of the free carboxyl group of this diselenide derivative. Typical dark-field microscopic images of the H-SeCyst-OD/PC liposomes by a 
high-resolution scanning transmission electron microscope (ARM-200CF, JEOL Ltd.) were shown in Fig. 2S in Supplementary materials.

The zeta-potential of the diselenide-free PC liposome was nearly zero due to the electrically neutral head groups of the PC molecules, while those of the diselenide / PC liposomes were dependent on the ionic property of the diselenide derivatives added. The values for H-SeCyst-OD/PC, Ac-SeCyst-OD / PC, D-SeCyst-OMe/PC and D-SeCyst-OH / PC liposomes were $13.6 \pm 6.17,-23.3 \pm 2.85,-7.92 \pm 2.38$ and $-34.5 \pm 4.50 \mathrm{mV}$, respectively. This trend was thought to reflect the chemical property of the diselenide derivatives, which suggested that the diselenide moiety was placed around the outer surface, not in the hydrophobic interior, of the liposomal membrane structures. Such an outer surface structure allows accessing of the peroxide substrates and the reducing substrate GSH to the diselenide moiety.

To estimate the actual chemical compositions of the obtained liposomes, the concentrations of selenium from the diselenides and phosphorus from PC in the liposome solutions were determined (Table 2). The actual compositions of the H-SeCyst-OD/PC and D-SeCyst-OH/PC liposomes with ionizable groups were $14.4 \pm 5.5$ and $16.8 \pm 4.55 \mathrm{~mol} \%$, respectively. Both $\mathrm{H}-\mathrm{SeCyst-OD}$ and D-SeCyst-OHwere favorably incorporated into the PC liposomal membranes. On the other hand, the actual compositions of Ac-SeCyst-OD and D-SeCyst-OMe with non-ionized (less polar) groups were, to some extent, lower than the calculated values $(20 \mathrm{~mol} \%)$. These two derivatives appeared to be less membrane-compatible than the H-SeCyst-OD and D-SeCyst-OH. Taken these results of the structural characterization of the liposomes together, the diselenide derivatives were thought to locate on the outer surface of the liposomal membranes that allow accessing the peroxide substrates and the reducing substrate GSH.

Several selenoproteins are reductive enzymes that operate through the reversible formation of selenenylsulfide bonds. The value for the redox potential of a selenenylsulfide bond in proteins was predicted to be within the range between the redox potentials of the disulfide and diselenide bonds [19]. Theoretically, the low redox potential of the diselenide bond may require a strong reducing agent, such as dithiothreitol, since the redox potential of 
SeCyst was found to be $-383 \mathrm{mV}$ [20]. Inside the living cell, key compounds responsible for maintaining disulfides in the reduced form are thioredoxin (Trx) and GSH, however, their redox potentials are $-270 \mathrm{mV}$ [21] and $-240 \mathrm{mV}$ [22], may not be sufficient for reduction of the diselenides. Metanis et al. reported that the redox potential of diselenide in a chemically synthesized glutaredoxin 3 (Grx3) mutant (Cys11Sec and Cys14Sec) was -309 mV [23]. The diselenide bond of the Grx3 mutant was actually cleaved by Trx. This Grx3 mutant further provided faster kinetics than the Grx mutants that contain a single Sec [23,24]. These facts suggest that diselenide-containing compounds have a potentially unique property in reductive catalysis. In this study, the GPx-like catalytic activity of the diselenide/PC liposomes was determined by the conventional NADPH method using the reducing substrate GSH (Fig. 3). Inorganic hydrogen peroxide and organic tert-butyl hydroperoxide and cumene hydroperoxide were used as the substrates. The diselenide free PC liposome did not show any GPx-like catalytic activity for the three peroxides. It is important to examine the effect of a functional site sulfur/selenium substitution on their redox potential and kinetics. As another control material, the disulfide analogues, which were substituted by sulfur in place of selenium in the diselenide derivatives, was similarly synthesized using L-Cyst as the starting material. The disulfide / PC liposomes also did not show the GPx-like catalytic activity for the three substrates (data not shown). The diselenide/PC liposomes showed the GPx-like catalytic activity for the three substrates in the order of hydrogen peroxide $>$ cumene hydroperoxide $>$ tert-butyl hydroperoxide. Such a trend in the activity may be due to the difference in the accessibility to the liposomal membrane scaffold among the three substrates. The H-SeCyst-OD/PC liposome exerted a higher GPx-like catalytic activity than the other diselenide/PC liposomes. This would be attributed to the facts that H-SeCyst-OD with the free $\alpha$-amino groups can be electrostatically attracted to the reducing substrate GSH possessing a negative character. Both the Ac-SeCyst-OD / PC and D-SeCyst-OMe / PC liposomes with the nonionized group showed a lower GPx-like catalytic activity. The D-SeCyst-OH / PC liposome was even less active than the Ac-SeCyst-OD / PC and D-SeCyst-OMe/PC liposomes. This may be explained by the fact that the D-SeCyst-OH with free $\alpha$-carboxyl groups is less accessible for the GSH molecule, because of its anionic character. 
In conclusion, we first synthesized the selenoprotein L-inspired membrane-compatible diselenide derivatives as a functional element of the GPx nano-mimics. Second, the diselenide derivatives were successfully incorporated into the phospholipid-based liposomal membrane scaffold. A cationic diselenide derivative in the GPx nano-mimics resulted in a higher GPx-like catalytic activity in comparison to the electrically neutral and anionic derivatives. Overall, the diselenide derivatives in a liposomal colloidal scaffold could possibly act as the GPx nano-mimics in physiological aqueous media. 


\section{References}

[1] K. Schwarz, C.M. Foltz, Selenium as an integral part of factor 3 against dietary necrotic liver degeneration, J. Am. Chem. Soc. 79 (1957) 3292-3293.

[2] M.J. Berry, L. Banu, Y. Chen, S.K. Mandel, J.D. Kieffer, J.W. Harney, P.R. Larsen, Recognition of UGA as a selenocysteine codon in Type I deiodinase requires sequences in the 3' untranslated region, Nature 353 (1991) 273-276.

[3] R. Walczak, E. Westhof, P. Carbon, A. Krol, A novel RNA structural motif in the selenocysteine insertion element of eukaryotic selenoprotein mRNAs, RNA 2 (1996) $367-379$.

[4] V.G. Kryukov, S. Castellrano, S.V. Novoselov, A.V. Labanov, O. Zehtab, R. Guigo, V. Gladyshev, Characterization of mammalian selenoproteomes, Science 300 (2003) $1439-1443$.

[5] T.L. Hendrickson, Selenium content in selected foods from the Greek market and estimation of the daily intake, Nat. Struct. Mol. Biol. 14 (2007) 100-101.

[6] C. Jacob, G.I. Giles, N.M. Giles, H. Sies, Sulfur and selenium - The role of oxidation state in protein structure and function, Angew. Chem. Int. Ed. Engl. 42 (2003) $4742-4758$.

[7] L. Johansson, G. Gafvelin, E.S. Arnér, Biochim. Biophys. Acta 1726 (2005) 1-13.

[8] N. Metanis, E. Keinan, P.E. Dawson, J. Am. Chem. Soc. 128 (2006) 16684-16691.

[9] A.V. Lobanov, D.L. Hatfield, V.N. Gladyshev, Eukaryotic selenoproteins and selenoproteomes, Biochim. Biophys. Acta 1790 (2009) 1424-1428.

[10] V.A. Shchedrina, S.V. Novoselov, M.Y. Malinouski, V.N. Gladyshev, Identification and characterization of a selenoprotein family containing a diselenide bond in a redox motif, Proc. Natl. Acad. Sci. U. S. A. 104 (2007) 13919-13924.

[11] M. Haratake, S. Matsumoto, M. Ono, M. Nakayama, Nanoparticulate glutathione peroxidase mimics based on selenocystine-pullulan conjugates, Bioconjugate Chem. 19 (2008) 1831-1839.

[12] M. Haratake, Y. Tachibana, Y. Emaya, S. Yoshida, T. Fuchigami, M. Nakayama, Synthesis of nanovesicular glutathione peroxidase mimics with a 
selenenylsulfide-bearing lipid, ACS Omega 1 (2016) 58-65.

[13] R.E. Kitson, M.G. Mellon, Colorimetric determination of phosphorus as molybdivanadophosphoric acid, Ind. Eng. Chem. Anal. Ed. 16 (1944) 379-383.

[14] J.H. Walkinson, Fluorometric determination of selenium in biological material with 2,3-diaminonaphthalene, Anal. Chem. 38 (1966) 92-97.

[15] S. Yoshida, E. Hori, S. Ura, M. Haratake, T. Fuchigami, M. Nakayama, A comprehensive analysis of selenium-binding proteins in the brain using its reactive metabolite, Chem. Pharm. Bull. 64 (2016) 52-58.

[16] M. Haratake, T. Sakano, T. Fuchigami, M. Nakayama, Thiol-targeted introduction of selenocysteine to polypeptides for synthesis of glutathione peroxidase mimics, Metallomics 3 (2011) 702-709.

[17] B. Mannervik, Glutathione peroxidase, Methods Enzymol. 113 (1985) 490-495.

[18] S.R. Wilson, A.P. Zucker, R.R.C. Huang, A. Spector, Development of synthetic compounds with glutathione peroxidase activity, J. Am. Chem. Soc. 111 (1989) $5936-5939$.

[19] T.C. Stadtman, Selenocysteine, Annu. Rev. Biochem. 65 (1996) 83-100.

[20] T. Nauser, S. Dockheer, R. Kissner, W.H. Koppenol, Catalysis of electron transfer by selenocysteine, Biochemistry 45 (2006) 6038-6043.

[21] F. Åslund, K.D. Berndt, A. Holmgren, Redox potentials of glutaredoxins and other thiol-disulfide oxidoreductases of the thioredoxin superfamily determined by direct protein-protein redox equilibria, J. Biol. Chem. 272 (1997) 30780-30786.

[22] J. Rost, S. Rapoport, Reduction-potential of glutathione, Nature 201 (1964) 185.

[23] N. Metanis, E. Keinan, P.E. Dawson, Synthetic seleno-glutaredoxin 3 analogues are highly reducing oxidoreductases with enhanced catalytic efficiency, J. Am. Chem. Soc. 128 (2006) 16684-16691.

[24] S. Muller, H. Senn, B. Gsell, W. Vetter, C. Baron, A. Böck, The formation of diselenide bridges in proteins by incorporation of selenocysteine residues: biosynthesis and characterization of (Se)2-thioredoxin, Biochemistry 33 (1994) 3404-3412. 


\section{Table 1}

$\mathrm{X}$-ray photoelectron spectroscopy binding energies of selenium $3 \mathrm{p}$ and $3 \mathrm{~d}$ electrons in diselenide derivatives.

\begin{tabular}{llll}
\hline & \multicolumn{2}{l}{ Binding energy $(\mathrm{eV})$} & \\
\cline { 2 - 4 } Diselenide & Selenium 3p $\mathrm{p}_{3 / 2}$ & Selenium 3p $\mathrm{p}_{1 / 2}$ & Selenium 3d \\
\hline H-SeCyst-OD & 161.8 & 167.6 & 55.7 \\
Ac-SeCyst-OD & 161.8 & 167.7 & 55.9 \\
D-SeCyst-OMe & 162.1 & 167.8 & 56.0 \\
D-SeCyst-OH & 161.7 & 167.5 & 55.8 \\
SeCyst & 161.7 & 167.6 & 55.6 \\
\hline
\end{tabular}

\section{Table 2}

Characterization of diselenide / PC liposomes.

\begin{tabular}{|c|c|c|c|c|}
\hline \multirow{2}{*}{ Liposome } & \multirow{2}{*}{$\begin{array}{l}\text { Particle } \\
\text { diameter }^{\mathrm{a}} \\
(\mathrm{nm})\end{array}$} & \multirow{2}{*}{$\begin{array}{l}\text { Zeta- } \\
\text { potential }^{\mathrm{a}} \\
(\mathrm{mV})\end{array}$} & \multicolumn{2}{|c|}{ Composition $^{\mathrm{a}, \mathrm{b}}(\mathrm{mol} \%)$} \\
\hline & & & Diselenide & $\mathrm{PC}$ \\
\hline H-SeCyst-OD / PC & $136.0 \pm 17.1$ & $13.6 \pm 6.2$ & $14.4 \pm 5.5$ & $85.6 \pm 5.5$ \\
\hline Ac-SeCyst-OD / PC & $76.0 \pm 21.4$ & $-23.3 \pm 2.9$ & $10.3 \pm 5.2$ & $89.7 \pm 5.2$ \\
\hline D-SeCyst-OMe/PC & $73.3 \pm 14.1$ & $-8.0 \pm 2.4$ & $5.5 \pm 1.9$ & $94.5 \pm 1.9$ \\
\hline D-SeCyst-OH/PC & $44.1 \pm 7.7$ & $-34.5 \pm 4.5$ & $16.8 \pm 4.6$ & $83.2 \pm 4.6$ \\
\hline PC alone & $94.5 \pm 0.4$ & $1.0 \pm 1.0$ & $0.0 \pm 0.4$ & $100.0 \pm 0.0$ \\
\hline
\end{tabular}

${ }^{\mathrm{a}}$ Values are mean \pm standard deviation $(n=3) . \quad{ }^{\mathrm{b}}$ Summation of diselenide and PC contents in liposomes was defined as $100 \mathrm{~mol} \%$. 

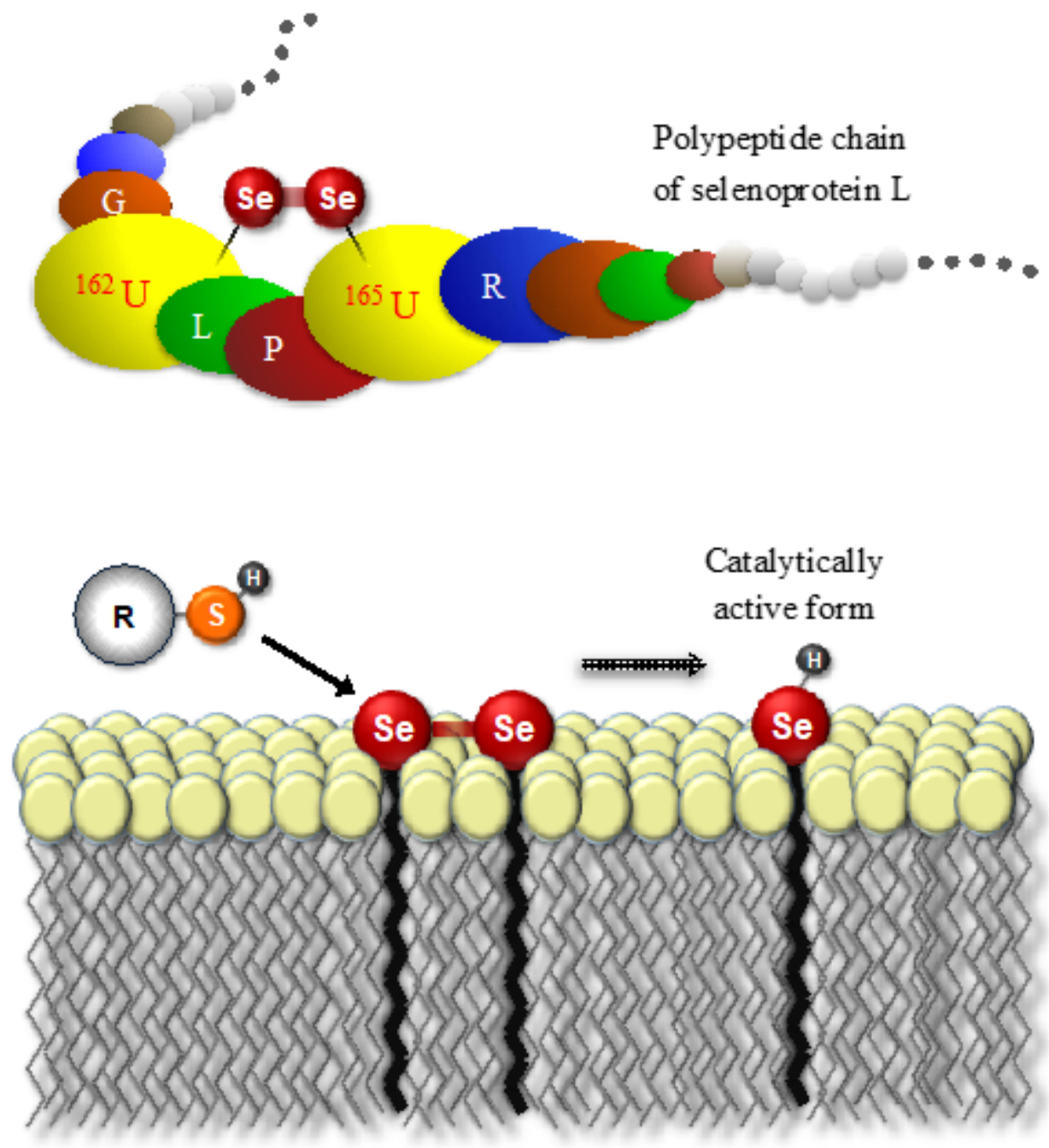

Fig. 1. Schematic drawings of intramolecular diselenide bond between ${ }^{162} U$ and ${ }^{165} U$ in the polypeptide chain of selenoprotein L (top) and diselenide derivative-containing liposome (bottom). U: Sec, SeCys residue, G: Gly, glycine residue, L: Leu, leucine residue, R: Arg, arginine residue. 

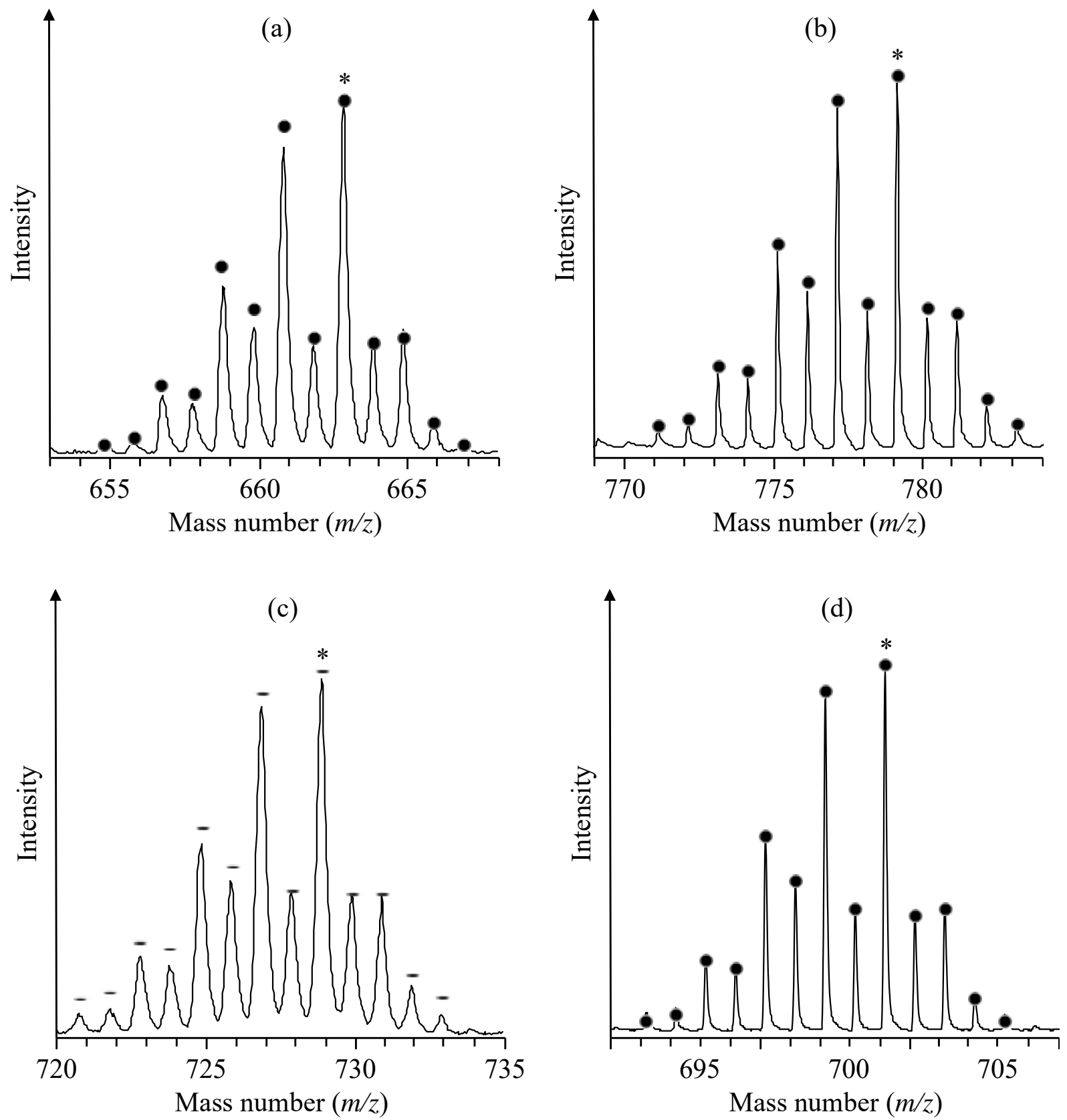

Fig. 2. Mass spectra of diselenide derivatives. (a) H-SeCyst-OD, (b) Ac-SeCyst-OD, (c) D-SeCyst-OMe, (d) D-SeCyst-OH. Asterisk indicates the peak of two ${ }^{80}$ Se atom-containing diselenide species. Solid ellipses indicate the calculated relative intensity of the diselenide stable isotope species. 


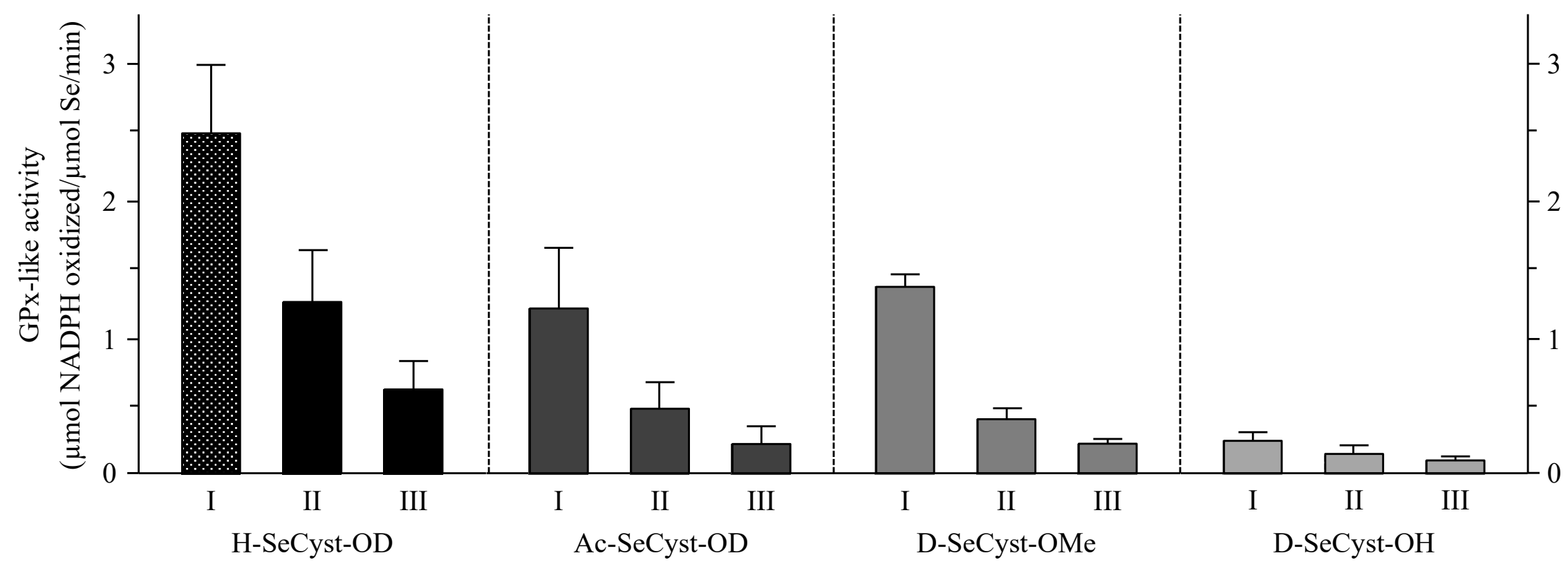

Fig. 3. GPx-like catalytic activity of diselenide/PC liposomes for inorganic and organic peroxide substrates. Peroxide substrate I: hydrogen peroxide, II: cumene hydroperoxide, III: tert-butyl hydroperoxide. Values are mean \pm standard error $(n=3)$. 
$[\overbrace{\mathrm{NH}_{2}}^{\mathrm{OH}} \mathrm{Se}]_{2}$
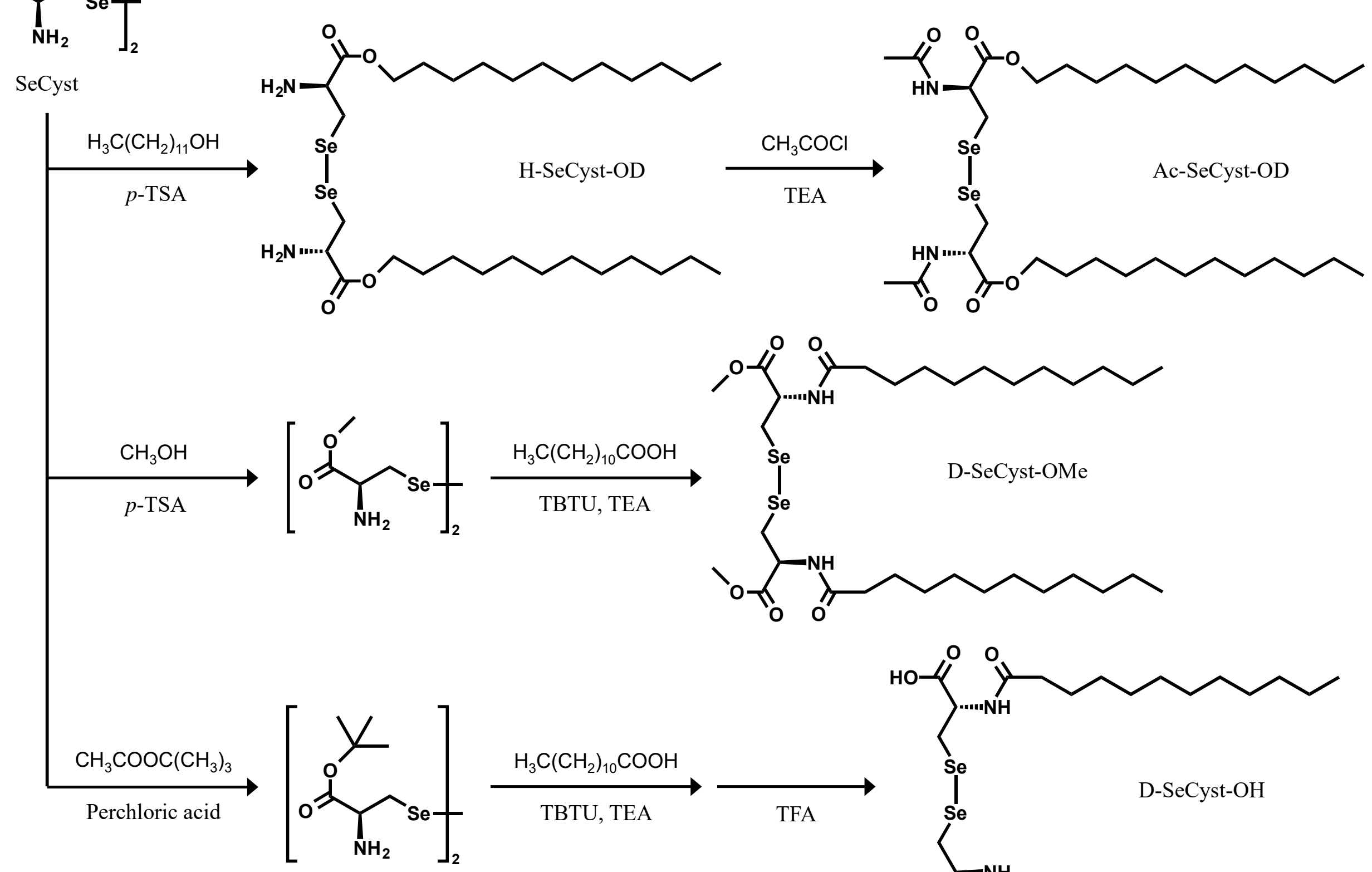